\title{
Lignes directrices de la Société suisse de psychiatrie d'assurance pour l'expertise médicale des troubles psychiques*
}

Société suisse de psychiatrie d'assurance, Association suisse d'experts médicaux pour les questions d'assurance en relation avec les troubles psychiques et psychosomatiques

Deutsch erschienen

in Nr. 20/2004
* Approuvé et mis en vigueur par l'assemblée générale de la Société suisse de psychiatrie d'assurance le 13 novembre 2003.

** Agency for Health-Care Policy and Research. Institution américaine élaborant les «Clinical Practice Guidelines».

\section{Introduction}

1. Définition du concept de ligne directrice L'AHCR $^{* *}$ définit comme suit le concept de ligne directrice: Les lignes directrices sont des propositions et recommandations développées de manière systématique dans le but d'aider patients et médecins à prendre, dans des situations cliniques précises, des décisions utiles du point de vue de la prévention, du diagnostic, du traitement et du suivi des maladies. Elles reflètent l'état des connaissances à un moment donné et doivent être périodiquement révisées. Elles prennent en compte les résultats d'études contrôlées, l'influence de la subjectivité des experts étant réduite à un minimum par le recours à des techniques comme les conférences de consensus, les processus Delphi ou la méthode du groupe nominal.

\section{Rôle des lignes directrices}

Les présentes lignes directrices sont un guide pour l'activité de l'expert. Elles reposent sur une revue des faits scientifiquement avérés et tiennent compte des expériences et des nécessités de la pratique. Leur formulation repose sur un large consensus entre experts. Elles ont le caractère de recommandations et non celui d'une norme légale contraignante.

\section{Domaine de validité des lignes directrices} Ces lignes directrices concernent les expertises demandées par les assurances dans les cas où se posent des questions d'ordre psychiatrique, psychosomatique et psychothérapeutique.

\section{Définition de l'expertise médicale}

Une expertise est définie au sens des présentes lignes directrices comme la prise de position d'un expert psychiatre indépendant dans le cadre d'un mandat et sous forme d'un rapport écrit répondant à des questions touchant à la psychiatrie d'assurance.

\section{Principes}

\section{Troubles psychiques}

Les troubles psychiques comportent des aspects biologiques, intrapsychiques et sociaux (cette dernière dimension incluant aussi les considérations d'ordre culturel et systémique), si bien que l'on parle aujourd'hui d'un modèle bio-psychosocial de la psychiatrie. Il appartient à l'expert de prendre en compte et de décrire tous ces différents aspects de la situation qui lui est soumise.

\section{Exigences juridiques}

Pour qu'un rapport médical ait valeur probante, il faut selon une jurisprudence constante du TFA que les points litigieux importants aient fait l'objet d'une étude fouillée, que le rapport se fonde sur des examens complets, qu'il prenne également en considération les plaintes exprimées par la personne examinée, qu'il ait été établi en pleine connaissance des dossiers antérieurs, que la description des tenants et aboutissants de la situation médicale et son appréciation soient plausibles et enfin que les conclusions de l'expert soient dûment motivées.

\section{L'expertise psychiatrique dans le contexte du droit des assurances}

L'expertise consiste d'abord en la présentation clinique d'un cas du point de vue psychiatrique. Le médecin doit prendre ici le rôle d'un expert dont la fonction est d'aider l'administration ou la justice dans un processus de prise de décision. La réponse aux questions d'ordre juridique qui lui sont posées exige une certaine familiarité avec le mode de pensée qui les sous-tend. La pratique du droit demande un mode de pensée normatif auquel le médecin va être tenté de s'adapter lorsqu'il sera mandaté comme expert. La science médicale repose pourtant davantage sur une approche empirique que sur une démarche normative. Le médecin travaillant dans le domaine des assurances demeure un clinicien appréhen- 
dant la réalité qui se présente à lui selon une logique médicale. Il doit présenter le résultat de ses investigations et son appréciation de la situation d'une manière propre à donner à l'instance qui l'a mandaté (généralement une assurance ou un tribunal) les informations dont elle a besoin pour rendre une décision, les différences entre épistémologie médicale et juridique nécessitant un processus de traduction des constatations médicales pour les adapter au cadre de réflexion juridique. Le fait que son mandataire attend des réponses claires formulées selon ses propres catégories de réflexion ne doit en l'occurrence pas amener l'expert à prendre des positions qui ne seraient plus conformes aux exigences de la science médicale. Mettre en lumière les limites du savoir médical et ne répondre le cas échéant que partiellement ou pas du tout aux questions posées demeure une tâche primordiale de la médecine d'assurance qui doit cultiver un dialogue constant avec les assureurs et les juristes en veillant au maintien de conditions permettant un exercice correct du "processus de traduction». Il s'agit notamment d'insister pour que les questions soumises soient pertinentes et de refuser que la responsabilité de répondre à des questions non-médicales ou pseudo-médicales soit transférée à l'expert.

La psychiatrie en tant que science médicale a ses limites et il importe que l'expert les souligne quand il se trouve confronté à des questions auxquelles il est impossible de répondre de façon suffisamment claire et précise.

\section{But de l'expertise psychiatrique}

L'expertise psychiatrique a pour but de déterminer un éventuel dommage à la santé d'un point de vue psychiatrique et dans une perspective médicale globale. Selon les circonstances particulières du mandat et la nature des questions posées, il s'agira d'apprécier les répercussions sur la capacité de travail de la personne examinée, d'émettre des propositions pour la suite du traitement et d'éventuelles mesures de réadaptation, d'évaluer un dommage permanent sous l'angle du droit à une rente ou à une indemnité pour atteinte à l'intégrité ou encore de donner au juriste les éléments nécessaires pour une prise de décision en matière de causalité.

\section{Objectivité et neutralité de l'expert}

L'expert doit occuper une position impartiale et neutre entre l'assurance et l'expertisé. Dans la mesure où il lui est impossible d'avoir avec la personne examinée le type de dialogue indispensable à la récolte des informations qui lui sont nécessaires sans établir avec elle une rela- tion d'empathie, il vaudrait mieux parler de «partialité multidirectionnelle» pour décrire l'attitude de l'expert. Il devrait par ailleurs rester objectif autant qu'on peut l'être dans le domaine de la psychiatrie, en s'efforçant constamment de prendre conscience et de se distancer des conceptions et motivations en relation avec son histoire et sa propre personne. L'entretien clinique constitue la principale méthode d'investigation du psychiatre. Il s'agit par définition d'un processus dialectique dont le résultat n'est pas déterminé que par l'expertisé mais aussi par l'expert. Le résultat de l'examen sera donc dans tous les cas marqué par des influences de la part de l'examinateur. La réflexion critique apportée par ce dernier à ses propres manières de penser, de ressentir et de se comporter constitue un critère de qualité primordial d'une expertise psychiatrique.

\section{Exigences posées à l'expert}

L'expert doit disposer d'une formation complète de spécialiste en psychiatrie et psychothérapie. Une familiarité avec la problématique et les concepts du droit des assurances est indispensable. L'expert doit constamment continuer à se former en psychiatrie et plus spécifiquement en médecine des assurances. Il devrait partager avec des collègues une réflexion à propos de sa pratique dans le cadre d'un cercle de qualité ou par des processus d'intervision/supervision. Il doit aussi s'intéresser de façon générale à des questions d'ordre culturel et social, plus particulièrement à celles touchant le monde du travail et le système des assurances.

\section{Le processus de I'expertise}

\section{Réalisation de l'expertise}

L'expert commence par réunir les informations pertinentes à partir de l'étude du dossier, des renseignements donnés par la personne examinée, de ceux éventuellement fournis par des tiers, de l'observation du tableau clinique et du comportement du sujet. Dans un deuxième temps, il s'efforce d'élaborer une représentation globale cohérente à partir de ces différents matériaux.

Tant qu'il n'y parvient pas d'une manière satisfaisante et qu'il n'est pas en mesure de répondre aux questions posées, l'expert essaie dans toute la mesure du possible de compléter et d'affiner sa perception de la situation en effectuant ou en demandant des investigations complémentaires ou en approfondissant sa réflexion. 


\section{Expertise psychiatrique et processus interpersonnel}

Lorsqu'il adopte une fonction d'expert, le praticien demeure médecin et reste en tant que tel soumis au principe fondamental du «primum nil nocere». Le déroulement de l'expertise va inévitablement avoir des répercussions sur l'état mental de la personne examinée. Quels que soient les efforts d'objectivité et de neutralité de l'examinateur, toute expertise médicale représente une intervention susceptible d'influer sur l'évolution de la maladie.

Il est par conséquent indispensable que l'expert prenne en compte dans sa réflexion la nature de l'interaction émotionnelle entre l'expertisé et lui-même, les facteurs motivationnels à l'œuvre chez l'expertisé de même que les questions liées aux mécanismes de défense, au transfert et au contre-transfert.

\section{Les différents niveaux d'appréciation de la qualité d'une expertise}

On peut évaluer en premier lieu la qualité de la récolte et de la description des informations. A un niveau supérieur, on appréciera la qualité de l'intégration des informations recueillies dans un tableau global cohérent, puis l'aspect convaincant et complet de la discussion et enfin la clarté et la précision de la réponse aux questions posées. Quand des questions restent ouvertes ou si des contradictions n'ont pas pu être résolues, l'expert devrait le mentionner explicitement.

\section{Facteurs externes}

Les facteurs suivants, qui ne dépendent pas de l'expert, peuvent influencer la qualité d'une expertise:

- Qualité de la documentation à disposition (Est-elle complète? A-t-elle été mise à jour récemment? Comprend-elle des renseignements quant à la nature et aux exigences de la place de travail et quant à la qualité de l'adaptation professionnelle dont l'expertisé a fait preuve jusque-là?);

- circonstances et motifs de la demande d'expertise;

- qualité des questions posées;

- qualité de la collaboration triangulaire assurance - avocat de la personne examinée expert;

- niveau d'information, de motivation et de coopération de l'expertisé;

- qualité de la collaboration des proches de la personne examinée, de ses médecins traitants, de son employeur, etc.

\section{Partie pratique}

1. Organisation et équipement du cabinet, respectivement de l'institution

Les locaux doivent permettre le déroulement de l'examen dans un cadre calme et agréable. Les rendez-vous doivent être communiqués de façon claire et l'expert doit lui-même les respecter avec ponctualité.

\section{Etude des dossiers}

Une bonne connaissance du dossier est importante pour l'appréciation puisqu'elle permet à l'expert de se représenter l'évolution diachronique de la situation et de faire le point des avis déjà exprimés qu'il aura à commenter dans sa discussion.

\section{Informations à la personne examinée}

L'expertisé doit être clairement informé dès le départ que l'examen a lieu dans le cadre d'une expertise médicale. Il doit savoir que l'expert a l'intention de se forger un avis indépendant, qu'il doit répondre aux questions qui lui sont posées et qu'il est dès lors délié du secret professionnel à l'égard de l'instance qui l'a mandaté. L'expertisé devrait si possible être informé de manière appropriée du résultat de l'expertise.

\section{Examen}

L'expertisé doit être examiné personnellement par l'expert. L'examen clinique comprenant la prise de l'anamnèse, l'étude de la sémiologie et l'observation du comportement constitue la partie centrale de l'expertise. L'évolution de la relation interpersonnelle doit faire l'objet d'une attention particulière (cf. également ci-dessus sous III.2.)

L'ampleur des investigations sera fonction des particularités du cas et de la nature des questions posées.

Comme déjà mentionné sous III.2., la qualité $\mathrm{du}$ processus d'expertise dépend aussi de la motivation et de la collaboration de la personne examinée. Outre ses déclarations, le tableau clinique et le comportement observés et la comparaison avec les renseignements objectifs provenant notamment du dossier ont un caractère déterminant.

\section{Relation avec l'évaluation somatique}

L'appréciation des aspects somatiques du tableau précède généralement dans le temps l'évaluation psychiatrique. Il est particulièrement important de disposer d'un bilan somatique de qualité lorsqu'on doit se prononcer dans des cas de troubles psychosomatiques puisque plusieurs diagnostics 
du chapitre F de la CIM-10 ne peuvent être posés qu'après exclusion d'une origine somatique des symptômes.

L'examen somatique et l'examen psychiatrique devraient être pratiqués de façon séparée par les spécialistes respectifs. Il n'est pas rare cependant que ce soit le dialogue interdisciplinaire qui permette de dégager de la manière la plus claire la signification d'un tableau clinique particulier.

\section{Informations apportées par des tiers}

Il peut être important de savoir comment la personne examinée est perçue dans son environnement familial et professionnel. Des renseignements ne peuvent cependant être recueillis auprès de tiers qu'avec l'autorisation de l'expertisé. Des contacts avec le médecin traitant (qu'il soit généraliste ou psychiatre) peuvent apporter de précieux renseignements à propos des antécédents médicaux et de la personnalité de l'expertisé, de sa collaboration dans le cadre du traitement, de la qualité de son entourage ainsi que pour l'élaboration de propositions thérapeutiques. Des informations apportées par des tiers peuvent ainsi constituer une partie importante de l'expertise mais ne doivent pas obligatoirement y figurer dans tous les cas. Les personnes interrogées doivent être informées quant à la nature de la démarche de manière analogue à ce qui a été mentionné sous IV.3

\section{Examens complémentaires}

Des tests psychologiques peuvent représenter un complément de l'investigation. L'examen clinique et sa mise en parallèle avec l'anamnèse gardent cependant un caractère déterminant.

Une documentation standardisée de la sémiologie psychiatrique selon des échelles particulières comme par exemple le système AMDP est également à considérer au plus comme un complément de l'examen clinique.

Des examens de laboratoire et d'autres examens paracliniques peuvent s'avérer indiqués dans certains cas.

\section{Rédaction du rapport d'expertise}

Le rapport d'expertise doit être clairement structuré. La source des informations qui y figurent doit être indiquée. Il doit être rédigé dans un langage clair et compréhensible par le juriste.

Le rapport ne doit pas être plus long que nécessaire. On évitera dans un souci de cohérence et de lisibilité les descriptions trop détaillées et les redondances.

Il doit comprendre les chapitres suivants, éventuellement subdivisés en sections:
- introduction;

- anamnèse et renseignements tirés du dossier;

- indications subjectives de l'expertisé;

- observation et autres résultats d'examens;

- discussion et réponse aux questions.

\section{Discussion}

La discussion repose sur l'intégration des renseignements issus du dossier, de l'anamnèse, des indications subjectives, de l'observation clinique et du résultat des examens complémentaires éventuellement pratiqués. La démarche intellectuelle de l'expert devrait en l'occurrence être présentée de manière compréhensible et les désaccords avec des évaluations antérieures devraient être commentés et fondés. L'expert devra le cas échéant mettre en lumière et expliquer les discordances entre les indications de l'expertisé et le tableau clinique observé. Il veillera aussi à mentionner les zones d'incertitude et les points contradictoires.

\section{Diagnostic}

On utilisera par principe pour le diagnostic des troubles psychiques les classifications ICD-10 ou DSM-IV. Le diagnostic sera si possible complété par des réflexions d'ordre étiologique ou d'autres précisions.

Le diagnostic de personnalité, la description de la psychodynamique, l'évaluation de l'influence de facteurs d'ordre notamment social sur l'évolution ainsi que l'appréciation du degré de gravité du tableau constituent également des points importants.

\section{Incapacité de travail}

L'incapacité de travail ne découle pas de façon immédiate du diagnostic, elle s'apprécie sur la base du type et de l'importance des limitations fonctionnelles causées par les troubles, les possibilités de compensation étant à prendre en compte de façon appropriée.

La capacité de travail sera indiquée en pourcent ou en heures par jour. Quand on indique une capacité de travail sur le mode horaire, il faut préciser la nature des limitations additionnelles que peuvent constituer la nécessité de faire des pauses, un rendement réduit, une flexibilité diminuée. L'expert doit ici encore développer les raisons motivant ses conclusions.

\section{Réponse aux questions}

L'expert doit prendre en compte dans la discussion toutes les questions qui lui sont posées mais il ne doit y répondre que dans la mesure où cela lui est possible sur la base de ses connaissances médicales. 


\section{Réadaptation professionnelle}

Le succès des mesures de réadaptation professionnelle que l'expert sera éventuellement appelé à proposer ne dépend pas que de leur caractère approprié mais aussi, entre autres, des compétences et de l'engagement qui pourront être investis de la part de l'assureur, (notamment par le «case manager» responsable du suivi systématique du cas) de la motivation de l'expertisé et du soutien qu'il recevra de la part de son entourage.

\section{Examen de personnes de langue étrangère}

Quand le niveau de compréhension linguistique entre l'expert et la personne examinée n'est pas suffisant, on aura recours à un interprète qualifié.

\section{Expertises interdisciplinaires}

Ce n'est pas la juxtaposition d'une collection d'expertises produites par des spécialistes de différentes disciplines mais le dialogue entre les différents spécialistes impliqués qui permettra de dégager une vue d'ensemble satisfaisante. L'importance et l'étendue de la partie psychiatrique d'une expertise interdisciplinaire s'orientera en fonction de sa portée dans le cas particulier.

\section{Catamnèse}

Toute activité scientifique demande à celui qui l'exerce d'étendre ses connaissances et de perfectionner sa pratique en fonction des résultats concrets de son travail. Il est donc souhaitable que les experts reçoivent plus souvent que ce n'est actuellement le cas un feed-back de la part des instances qui les ont mandatés. 